\title{
Bi-Level Model for Public Rail Transportation under Incomplete Data
}

\author{
Kristina Pavlova, Todor Stoilov, Krasimira Stoilova
}

Institute of Information and Communication Technologies, Bulgarian Academy of Sciences, 1113 Sofia, Bulgaria

E-mails: kristina@hsi.iccs.bas.bg todor@hsi.iccs.bas.bg k.stoilova@hsi.iccs.bas.bg

\begin{abstract}
The increase of the utilization of public rail transportations is searched in directions for redistribution of the passenger travels between rail and bus transportation. The rail transport benefits by redistribution of the transportation flows on paths, predominantly supported by rails. The redistribution of the transportation is formalized by bi-level optimization problem. The upper level optimization estimates the maximal flow, which can be transported through a transportation network, supported both by bus and rail transports. The lower level optimization gives priority to the rail transport by decreasing the costs of flow distribution, using rail transport. This bi-level optimization problem was applied for the case of optimization of the rail exploitation in Bulgaria, defining priorities in transportation of the National transport scheme.
\end{abstract}

Keywords: Bi-level optimization, rail transportation, max-flow problem, network design.

\section{Introduction}

The public rail transportation is an important player for the development of Intelligent Transportation Systems. The rail transportation is giving advantages due to its benefits, concerning efficiency in exploitation, improving logistics services, reducing the pollution. This paper tries to give priority to rail transport in comparison with the bus transport in relation with the project with "BDZ Passenger Services", Ltd., in Bulgaria and project on a Program for career development of young scientists, funded by Bulgarian Academy of Sciences. The problems, which are defined and solved, are using data coming from the current practice in Bulgaria, related with the passenger transportation by both rail and buses. Due to the lack of full set of data about the intensity of the passenger transport, this research applies only available information from the schedules of buses and rail transport. To give priority to the rail transport is an important National policy for managing and implementation of intelligent transportation systems. 
A trivial approach for increase of rail transport is to consider and to provide bus transportation only on places where rail transportation is missing. However, capacity restrictions of the rail system, cross connections between bus and rail transportation routes, common stops for bus and rail in towns provide competitions between the rail and bus transportation services. In such competition environment, a special management policy is needed to give preferences and to increase the exploitation of the rail transport.

This paper tries to formalize the problem for increasing the priority of the rail transport. The formal description of this problem is to provide an optimal allocation of the rail transportation services over predefined transportation network, taking advantage from the bus transport. An optimization problem is defined, which generates a solution increasing the rail transport. The optimization problem is defined in a bi-level form. The upper optimization problem evaluates the maximal flow, which can pass between two predefined points of the transportation network. In the network the maximal flow is implemented both by bus and rail transport according to the available capacities of the transportation links.

On the lower level, the optimization problem gives preferences to the rail transport in comparison with the bus one. This priority is defined, applying low cost flow distribution through rail links. Thus, by minimization of the total costs of flow distribution the optimal solution will provide intensive utilization of rail links for the flow distribution, which will increase the rail utilization. This low level solution giving flow per link will give new values for the link capacities for the upper level problem. Thus, the two optimization problems are interconnected in bi-level optimization one. The later will give optimal solutions both by maximization of the transport flow between two predefined important nodes of the transportation network and redistribution of the maximal flow to links, supported by rail. Thus, predominant part of the passenger transport will be implemented by rail transport.

The paper makes an illustration of definition and solution of a transportation problem by bi-level optimization. The hierarchical order is applied for two optimization problems. The paper does not contain development of new algorithms for solution of bi-level problems, but it contributes for the increase of the application area of this more complex and advanced optimization formalism.

\section{Case study}

The idea for the bi-level definition of the problem about increase the rail utilization can be regarded as controversial problem or problem of synthesis, in comparison with the problem of analysis for evaluation of the maximal flow in a network. The problem for the evaluation of the maximal flow between two nodes, connected by a network was raised up in [14] by project RAND. This problem has been stated as follows: when the capacities of individual links in a rail network are given, how can be evaluated the over-all capability of the entire rail network. The researches in [14] target the evaluation of the maximal flow, which can be transported through a network. In [12] the minimal cut problem was defined in current form as maximal flow in a network. Now, it is well known that the maximal flow is equal to the 
minimal cut of the network. An extended history for the origins of this theoretical result can be found in [27].

The "maximal flow" problem can be assumed as a problem for analysis of a network system. The optimization problem can be defined in the form: a topology of a network with capacity values on the links of the network is given. How much is the maximal amount of the flow between two nodes, which can be passed through the network.

The analytical definition of the maximal flow problem is presented in the form

$$
\begin{gathered}
\max _{f_{i j}}\left[f_{s t}\right], \\
\sum_{j \in A(j)} f_{i j}-\sum_{j \in B(j)} f_{j i}=\left\{\begin{array}{c}
0, i \neq s, t, \\
f_{s t} *, i=s, \\
-f_{s t} *, i=t,
\end{array}\right. \\
f_{i j} \leq v_{i j},(\forall i, j) \in N,
\end{gathered}
$$

where $(i, j)$ denote the number of nodes in a network with $N$ nodes; $A(i)=\{j \in N\}$ is a set of nodes $i$, which origine incoming links to node $j$ (Fig. 1); $B(i)=\{j \in N\}$ is a set of nodes $i$, which are connected with the outgoing links from node $j$ (Fig. 1); $s$ and $t$ denote the source node and sink node; $v_{i j}$ are the capacities of the links between node $i$ and $j,(i, j) \in N ; f_{i j}$ are the unknown volumes of flows, which have to be evaluated as components of the maximal flow in the network.
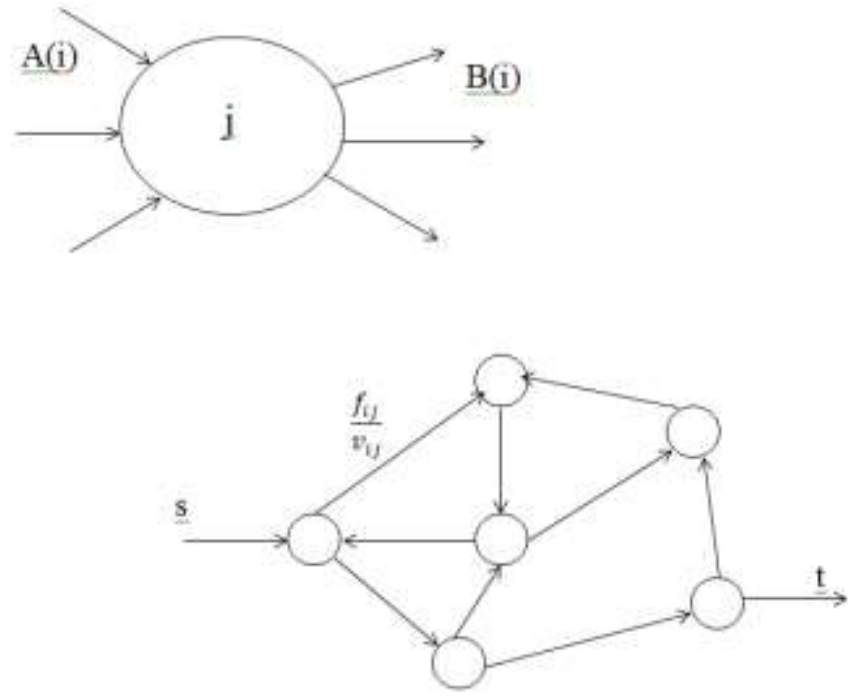

Fig. 1. Incoming and outgoing flows to/from node $j$, source $s$ and destination $t$ nodes

Relation (1b) describes the continuity nature of the flows, which enter and outgo to/from a node. Relation (1c) insists the flows on a link to respect the link capacity, which is predefined and constrained. The goal function (1a) describes the requirement for maximization of the flow $f_{s t}$, which starts from the initial node $s$ and enter to the final one $t$. 
The solution of problem (1) gives a value $f_{s t}$ of the maximal flow, which can be transmitted between $s$ and $t$ trough the network. Additionally, the values of $f_{i j}$ define the paths which the components of the maximal flow uses through the network.

Hence, for given capacity on the network links, the maximal flow problem (1) gives the amount of flow, which can pass through the predefined network topology.

Another corresponding optimization problem, defined as a problem for synthesis, provides minimization of the costs for flow distribution in the network for transportation of predefined value of traffic. Having optimal flow distribution, the corresponding values of the flow components $f_{i j}, i, j=1, \ldots, N$, can be used for evaluation of the minimal capacity $v_{i j}$ of each link $i, j$ or $v_{i j}=f_{i j}, i, j=1, \ldots, N$. The analytical form of the minimal cost distribution problem in linear form can be written as:

$$
\begin{gathered}
\min _{x_{i j}} \sum_{i j \in A} c_{i j} f_{i j}, \\
\sum_{j \in A(j)} f_{i j}-\sum_{j \in B(j)} f_{j i}=\left\{\begin{array}{c}
0, i \neq s, t, \\
f_{s t} *, i=s, \\
-f_{s t} *, i=t,
\end{array}\right. \\
a_{i j} \leq f_{i j} \leq b_{i j}, \quad i, j \in N,
\end{gathered}
$$

where $f_{i j}$ are the values of the flows of the links between nodes $i$ and $j$; $c_{i j}$ are the cost for transportation a unit flow between nodes $i$ and $j ; a_{i j}$ and $b_{i j}$ are the lower and upper boundaries of the flow between nodes $i$ and $j ; f_{s t}$ is the flow, which has to be transmitted from node $s$ to $t$ by minimizing the transportation costs (2a).

Relations (2b) correspond to the equations for flow conservation. The solution of (2) gives optimal flow distribution $f_{i j}$. The values of $f_{i j}$ define the minimal capacities $v_{i j}$ of the links by means to provide the cheapest flow distribution. A verification of problem (2) related to the design of network topology is introduced by [13].

This paper makes an integration of the both optimization problems (1) and (2). Problem (1) defines the maximal flow and the links used for its transportation, respecting the given set of links' capacities $v_{i j}$. Problem (2) will give an optimal flow distribution $f_{i j}$, giving priority to the rail transportation. This flow distribution defines the minimal link capacities $v_{i j}=f_{i j}$ of the network. The integration of (1) and (2) makes a synthesis for a network topology, where the value of flow between two nodes $s$ and $t$ is maximal and the components of the maximal flow predominantly passes through links, supported by rail. The integration of these two problems is performed by applying bi-level optimization model.

The graphical presentation of the integration of (1) and (2) by bi-level optimization problem is presented in Fig. 2. The bi-level problem simultaneously makes maximization of flows between two predefined nodes of the network and performs priority flow distribution through links, supported by rail transport. The priority to rail transport is implemented by making minimal cost flow distribution where the costs for rail transport are lower in comparison with the costs for bus transportation. The low cost flow distribution is used for estimation the link capacities in the transportation network. 
The solution of the upper level problem will evaluate the maximal flow $f_{s t}$ between nodes $s$ and $t$. The value $f_{s t}$ will be used as a parameter in the constraints of problem (2). Then the lower level problem (2) will evaluate the flow distribution $f_{i j}=v_{i j}$, which on its turn will change the constraints of problem (1). As a result, the bi-level problem will give optimal solution for both the maximal flow $f_{s t}$ and the flow distribution $f_{i j}$ with priority to rail transportation in the network. The bi-level formalization will provide maximization of the flow $f_{s t}$ and will minimize the cost for link flows.

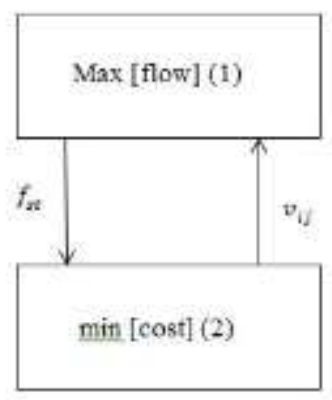

Fig. 2. Integration of optimization problems in bi-level optimization one

To identify peculiarities of the bi-level optimization model the next paragraph makes short overview of the current applications of the bi-level and multilevel hierarchical optimization.

\section{Bi-level optimization models and their applications}

The applications of bi-level optimization problems are a challenging formal approach for definition and solution of real world and practical problems nowadays. The idea of the bi-level formalism applies interconnections between two optimization problems. The upper optimization problem with its solution influences the parameters of the constraints and/or the goal function of the lower optimization problem. The lower one on its turn also influences by its solution the parameters of the constraints and/or the goal function of the upper level optimization. Such interconnected optimizations are formal instruments of the hierarchical system theory. However, these problems are difficult to be solved, because they insist application of solvers, which evaluate the global optimal solutions for non-convex cases. History about bilevel statement can be found in [31]. An extended analysis of the bi-level optimization can be seen in $[1,7,8]$. These papers pay attention and discuss the available application examples.

The short illustration about the applications of bi-level modeling and optimization is:

- Revenue management in economic systems. The main applications concern approaches for implementation of Stackelberg games [3, 4, 6, 20]. 
- Identification of parameters, control of transportation systems, scheduling $[17,18,28,29]$.

- Design of network topologies and facility location $[19,30]$.

- Optimal design of engineering problems [23, 32].

- Optimal exploitation in Energy sector [10,16].

- Gas pricing and distribution [9].

- Resource allocation $[11,15,33]$.

- Supply chain models [5, 21].

- Security issues [22].

This sequence of published cases with application of bi-level formalism tries to prove the conclusion that currently there is increasing interest for the implementation of the bi-level optimization in real cases and for solving real practical problems. Despite the internal complexity in solving bi-level optimization problems, the attempts for usage and application of such hierarchical optimization models are continuous because of the well-proved positive benefits.

\section{Graph presentation of the transport network between Sofia and Varna in Bulgaria}

The transport network was estimated for the real case between two important towns in Bulgaria: Sofia and Varna. The input data for the rail transportation from Sofia to Varna was taken from the real operating schedule, presented on-line in www.bdz.bg (Fig. 3).

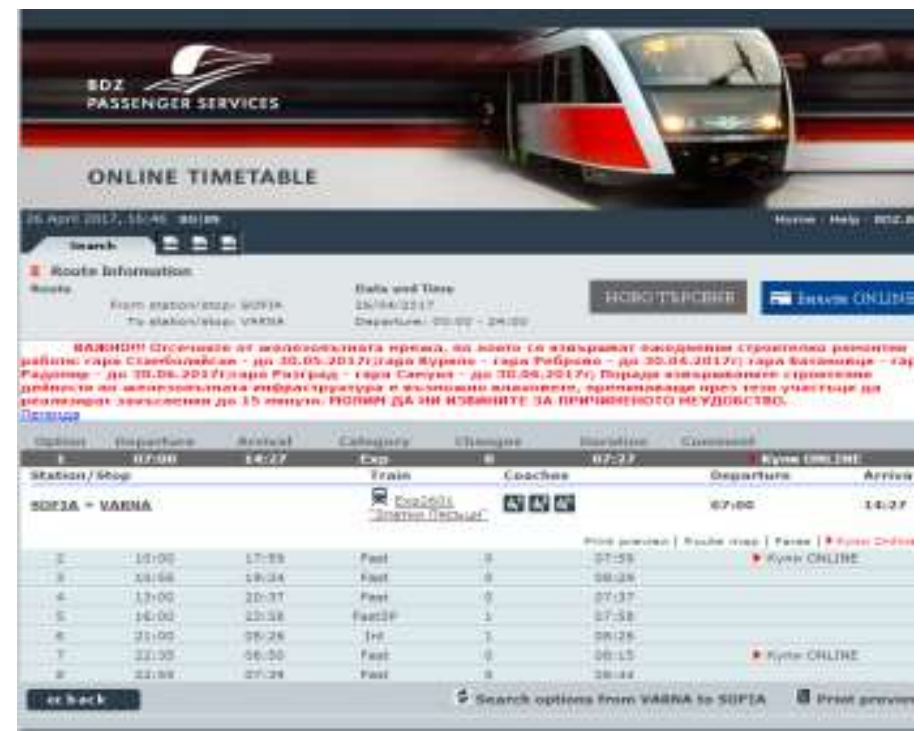

Fig. 3. The current schedule of trains between Sofia and Varna

Following the rail schedule it has been identified 17 stops, which define the network structure with 17 nodes. The actual lines for bus transportation were taken from the Ministry of Transport, Information Technology and Communications, 
which issues the licensees for bus transportation, publically available in http://rta.government.bg/index.php?page=scategories\&scategory=otrts.

All bus lines, which cross the rail lines of the direction Sofia-Varna, are included in the transportation network. The topology of the transportation network is created according to Fig.3 with initial node Sofia (number 1 or $s$ ) and final one - Varna (number 17 or $t$ ). The upper lines of the network correspond to the links of rail transportation. The others are undertaken by the bus transport (Fig. 4).

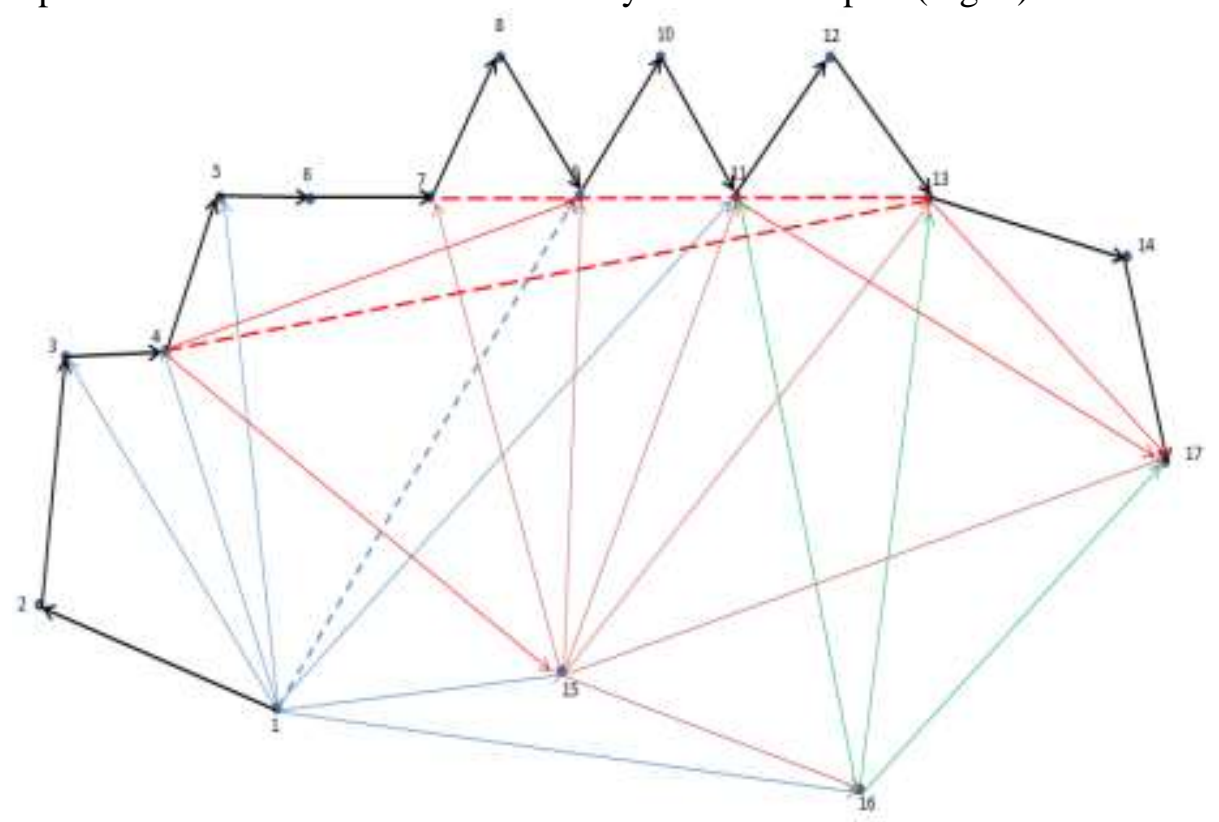

Fig. 4. Topology of the transportation network between Sofia and Varna

The transport capacities of each link must be estimated. Because data for the passenger flows are not available, an alternative way for evaluation of the flow capacities of the links is suggested for this research. It has been taking into consideration the duration of the transportation per different links of the network. The customers prefer their travel to be faster. Thus, the smaller time for traveling between couple of nodes gives more preferences for traveling on this direction by the customers. Additionally, the long travel time is a metric for low capacity for transportation per corresponding link. Thus, the transport capacities per link in the network are strongly related with the travel time per this link. For the current case, it has been chosen simple relation between the travel time $t_{i j}$ per link and the flow capacity $v_{i j}$ :

$$
v_{i j}=1 / t_{i j}, \quad i, j \in N .
$$

Relation (3) can be complicated with additional considerations, for example costs of travel. To simplify the numerical evaluations, this research applies relation (3). The numerical evaluations use the schedule of the trains (Fig. 5). 


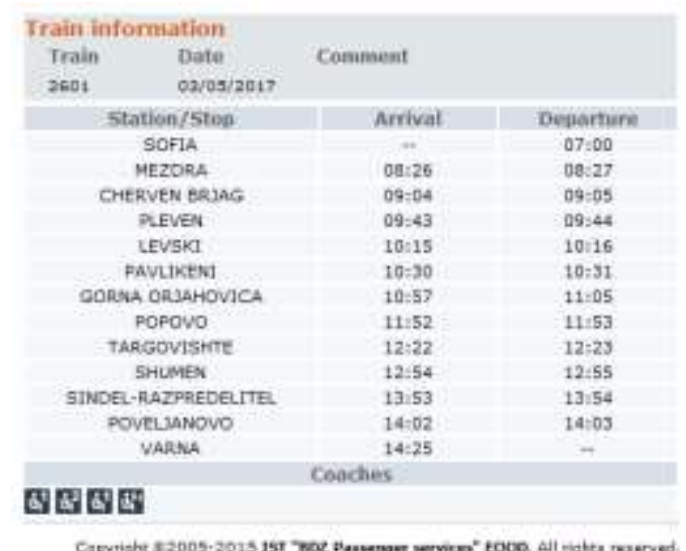

Fig. 5. Schedule of trains Sofia-Varna

The travel duration for the first two nodes in Fig. 5 - the link Sofia-Mezdra, is 1 hour and 26 minutes (86 minutes, from 7:00 until 8:26). Using (3) the link capacity for transportation between these two nodes of the network is: $1 / 86=0.011$ relative capacity units. Taking into account from Fig. 4 that 5 trains support this destination, the capacity for transportation on daily basis is: 5 trains $\times 0.011=0.055$ relative capacity units. This evaluation considers that the total capacity value is a sum of the individual capacities, performed by the different trains, according to their schedule.

Applying this manner of evaluation of the link capacity of daily trains schedule between Sofia and Varna, it has been identified the link capacities, given in Fig. 6. The first line presents the evaluated average capacity per link, used for rail travels and applied in the optimization problem.

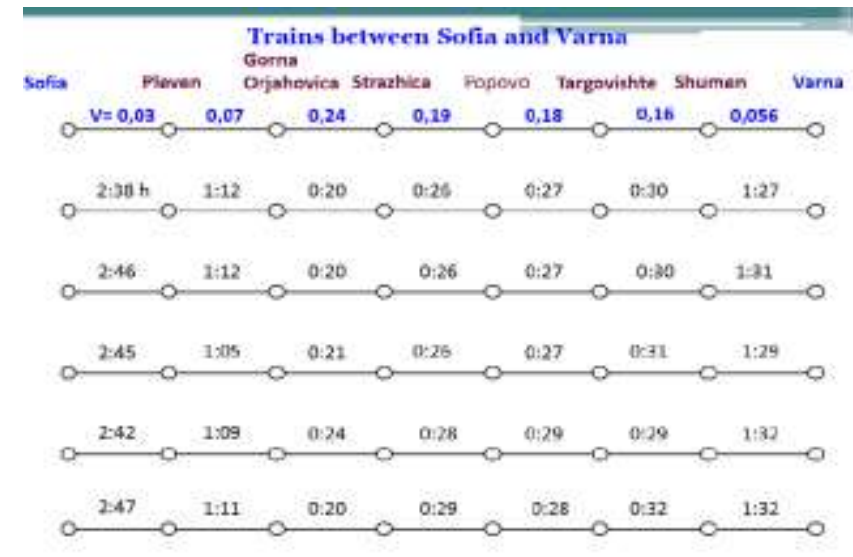

Fig. 6. Evaluation of the travel duration and capacities of links

The evaluation of the link capacities supported by bus transportation uses data given by their time schedules. These data are public available and they are presented as EXCEL files, illustrated on Fig. 7. 


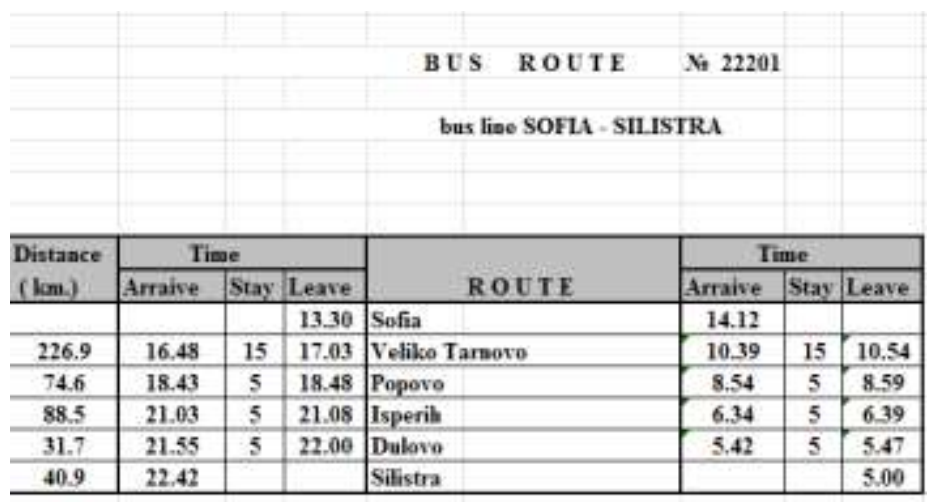

Fig. 7. Example for a bus schedule

For the case of Fig. 7 the bus schedule starts from Sofia but its destination differs from the final town Varna. Hence, the network links for this bus correspond only for the parts of the network, where the bus links cross the train links. For this case, this cross point is town of Popovo (line 3 on Fig. 7). This means that from Sofia to Popovo it exists two manners of transportations: by train and by bus. For the network topology, a link from Sofia to Popovo is added into the transport network, which is supported by the bus transport. The transport capacity of this link is: 5 hour and 18 minutes or 318 minutes (from 13:30 until 18:48) or the link capacity is $1 / 318$ relative units.

By integration of all capacities, which are estimated for the links, supported by buses, the average transportation time per link is given graphically on Fig. 8 .

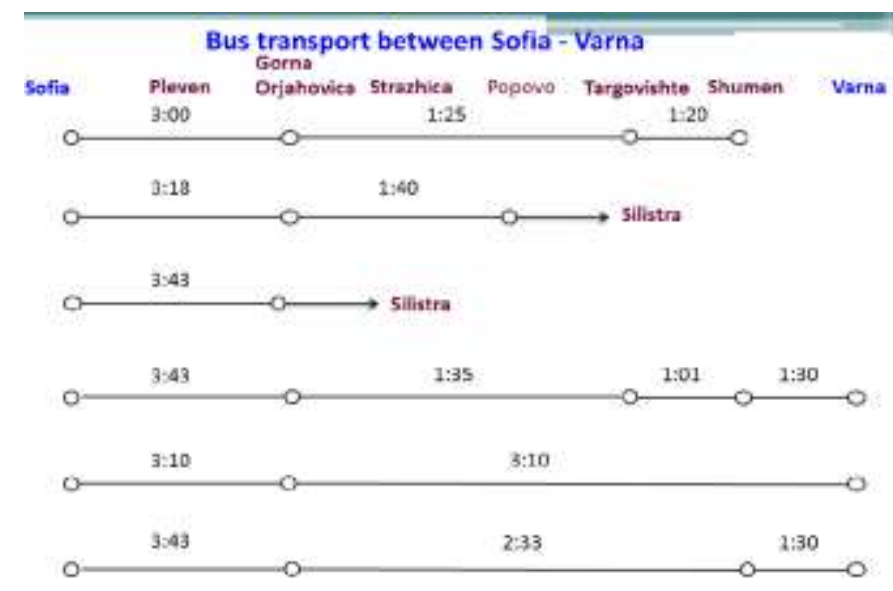

Fig. 8. Evaluation of the average travel time between nodes of the transportation network implemented by bus transport

These average values were obtained considering 44 bus lines and schedules between nodes, corresponding to common stops both for rail and bus transportations. The final estimation of the transport network and the capacities per link are presented graphically on Fig. 9. 


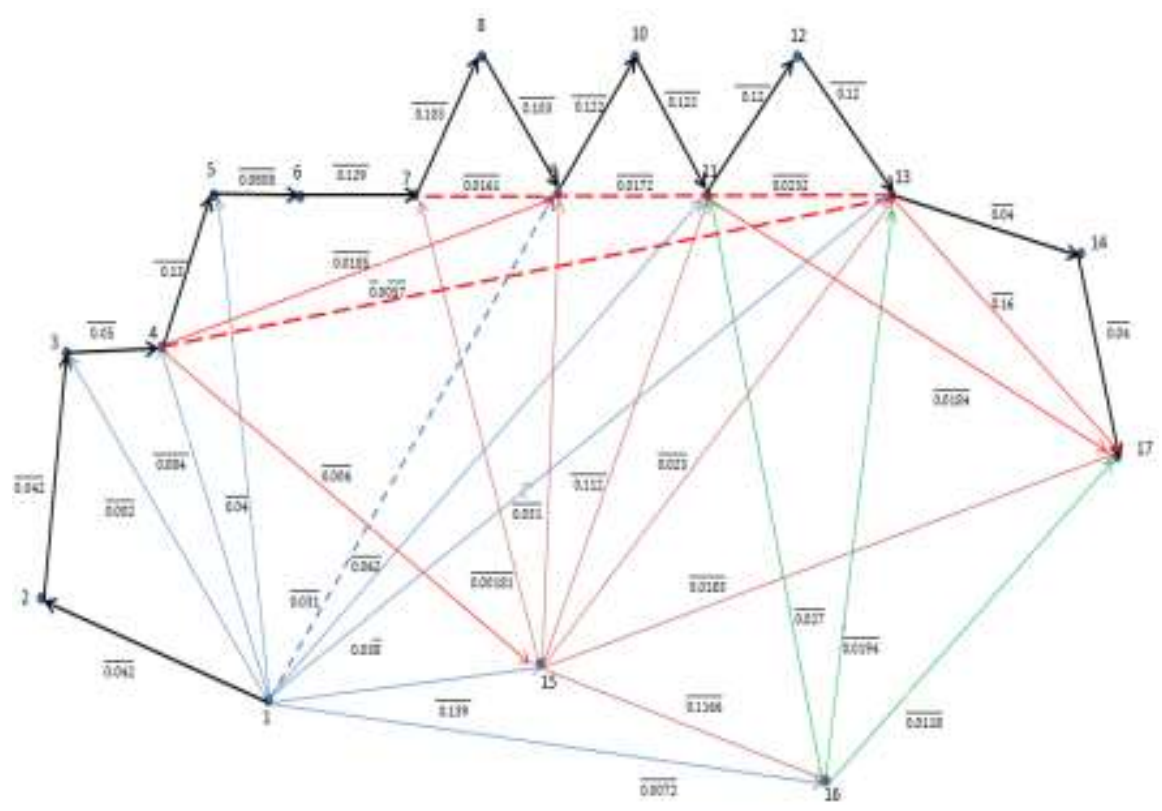

Fig. 9. Estimated link capacities

\section{Definition of the upper level optimization problem}

The analytical form of the maximal flow problem is (1). Following the network topology and the estimated parameters from Fig. 9, the analytical form of the "maxflow problem" is defined as a linear optimization problem. For each node $i$, $i=2, \ldots, 16$, of the network on Fig. 9 equilibrium relations for the flow continuity are defined. For nodes $1(s)$ and $17(t)$ the equilibrium equations contain the values of maximal flow $f_{s t}$, which is unknown value.

Example for equilibrium relation for node 5: $f_{4,5}+f_{1,5}-f_{5,6}=0, f_{i, j}$ is the flow between nodes $i$ and $j$.

Equilibrium relation for the source node $s=1$ :

$$
f_{1,2}+f_{1,3}+f_{1,4}+f_{1,5}+f_{1,9}+f_{1,11}+f_{1,13}+f_{1,15}+f_{1,16}-f_{s t}=0 .
$$

Equilibrium relation for the destination node $t=17$ :

$$
f_{14,17}+f_{13,17}+f_{11,17}+f_{15,17}+f_{16,17}-f_{s t}=0 .
$$

The flows have to respect constraints for the capacities of the links:

$$
f_{i, j} \leq v_{i, j}, \quad i, j=1, \ldots, N \text {. }
$$

The goal function of the max-flow problem is maximization of the value $f_{\mathrm{st}}$ :

$$
\max _{f_{s t}, f_{i j} i, j=1, N}\left(f_{s t}\right) \text {. }
$$

The optimization problem has 44 variables and 61 constraints, which contain 17 constraints for flow equilibrium for each of the 17 nodes and 44 upper bounds constraints. The problem has been solved, applying a MATLAB function graphmaxflow(). The solution of the optimization problem is presented graphically on Fig. 10. 


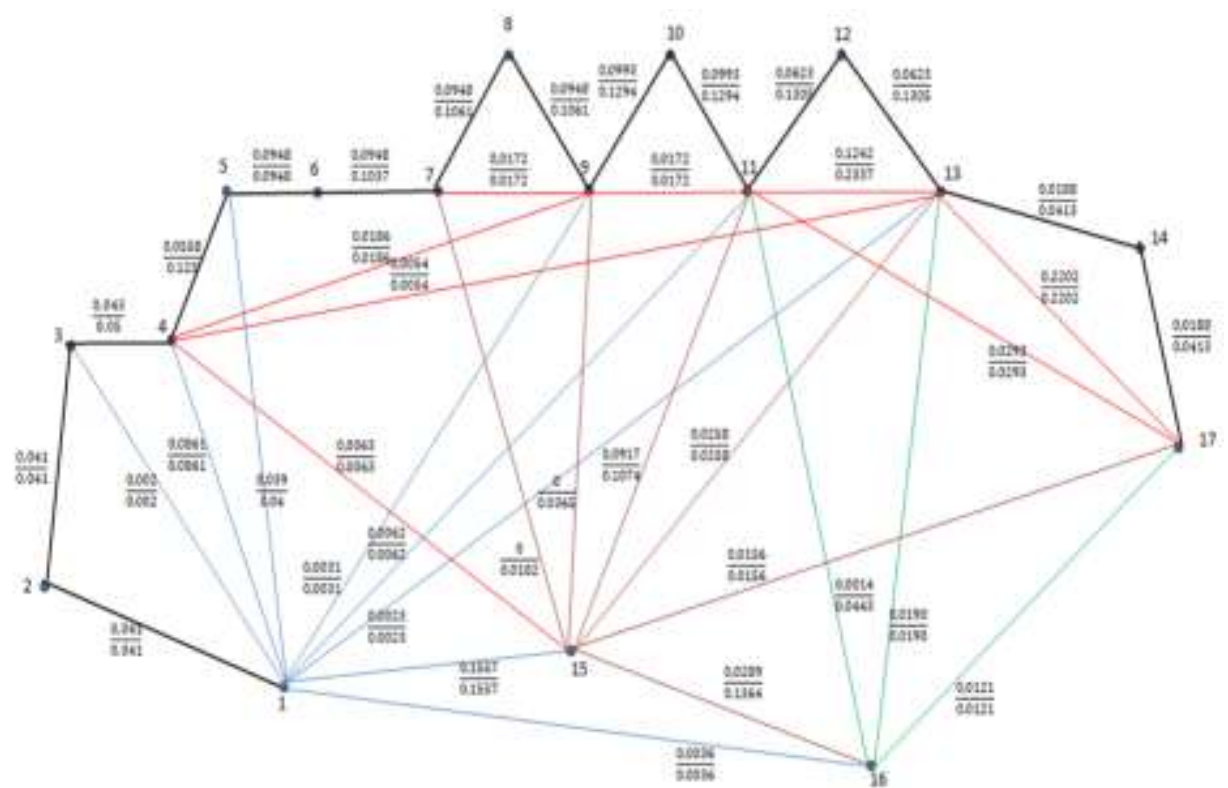

Fig. 10. Graphical presentation of "max-flow" problem solution

To each link of the transport network a fraction numbers is given. The numerator presents the value of flow, which is a part of the max-flow, passing through this link. The denominator is the capacity of the link:

$$
\frac{\text { numerator }}{\text { denominator }}=\frac{\text { component of max flow }}{\text { capacity of link }} \text {. }
$$

The solution of problem (1) gives value of the maximal flow, which can be transmitted from node 1 to node 17 (from Sofia to Varna). The value of this flow is 0.2485 relative flow units. Making comparisons for each link it can be identified, that the capacities of links between nodes 1-2, 2-3, supported by the rail are totally used by the components of the max flow. Hence, additional traffic between nodes 1 and 17 is not possible to be allocated. Respectively, this result proves decisions for the rail authorities to increase the capacities of rail transportation per these directions. This can be done by increasing the frequency of schedules for trains for these directions.

From Fig. 10 it is evident that a set of links supported by rail transportation are not used on their full capacities. This gives potential to redistribute the traffic flows by means to increase the rail utilization and to decrease the bus transport. Such policy can be implemented by definition and solution of additional optimization problem. The last will evaluate the cheapest flow distribution, taking into account the cost for rails lower than the cost for bus transportation. The flow distribution has to preserve capacity restrictions, defined in Fig. 9.

The optimization problem, which has to be solved, has the form (2). The particular case is that this problem has to respect the value of the maximal flow, which is estimated by the solution of problem (1). The requirement for low cost distribution on rail links will give priority to the rail transportation. The integration of these two problems is performed by bi-level optimization model. 


\section{Traffic flow distribution according to bi-level optimization model}

The bi-level optimization model follows the presentation of Fig. 6. The upper level problem evaluates the maximal flow, which can be transported between nodes 1 and 17. The components of max-flow respect the capacity constraints of the links, estimated according to Fig. 9. As a result, the value of the maximal flow is passed as a parameter to the lower optimization problem (2), defined as minimal cost flow distribution. The costs are defined in a way to give priority to the rail transport.

The minimal cost flow distribution problem evaluates the flows, which give preferences to the rail transport. These new values of flow distribution are passed to the upper optimization problem as new capacity restrictions for the max flow problem. The formal description of the bi-level problem is given in Fig. 11.

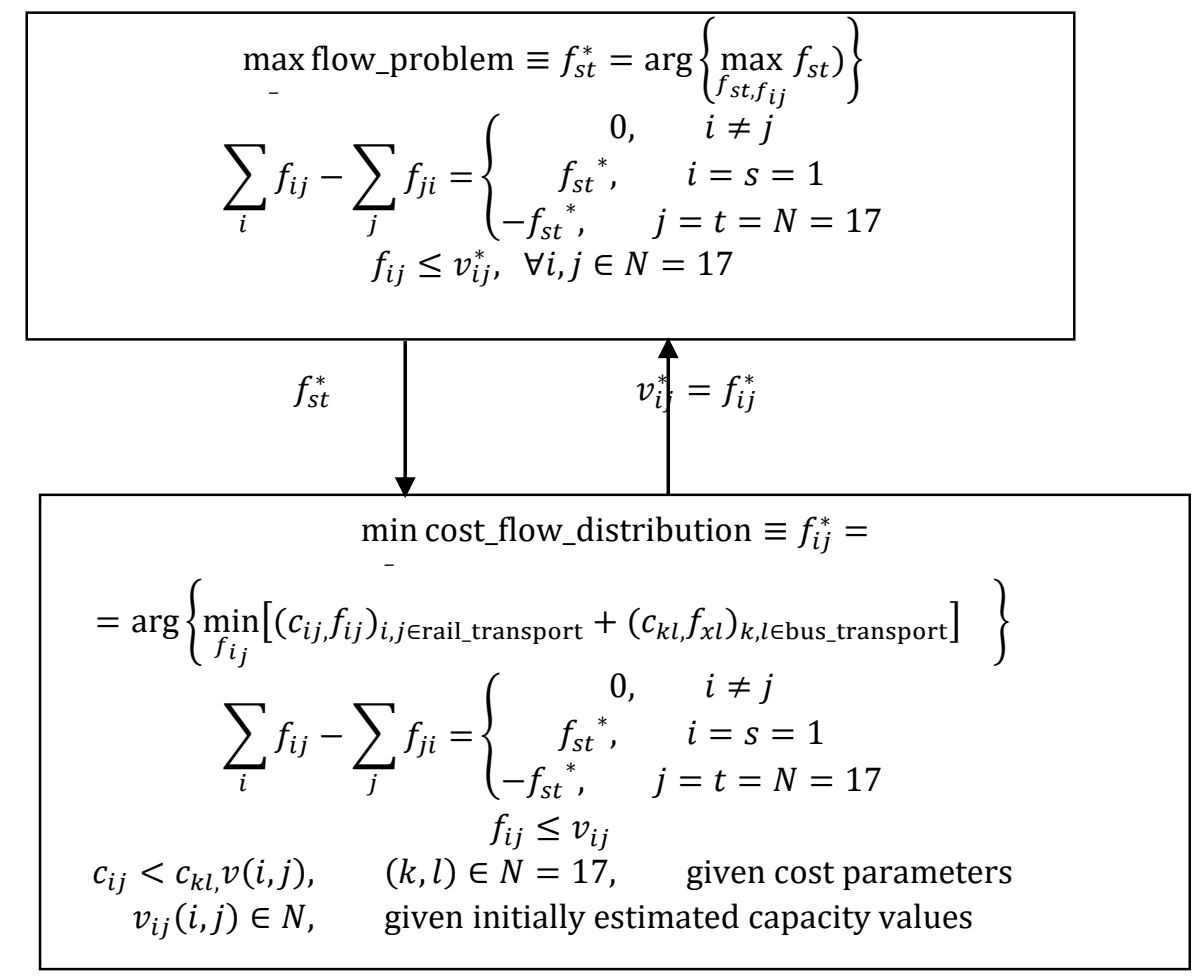

Fig. 11. Formal presentation of the bi-level problem

The solution of the bi-level problem is performed in MATLAB environment. The special function solvebilevel(), developed under the framework of project YALMIP [32] is applied. The bi-level solution is given on Fig. 12. 


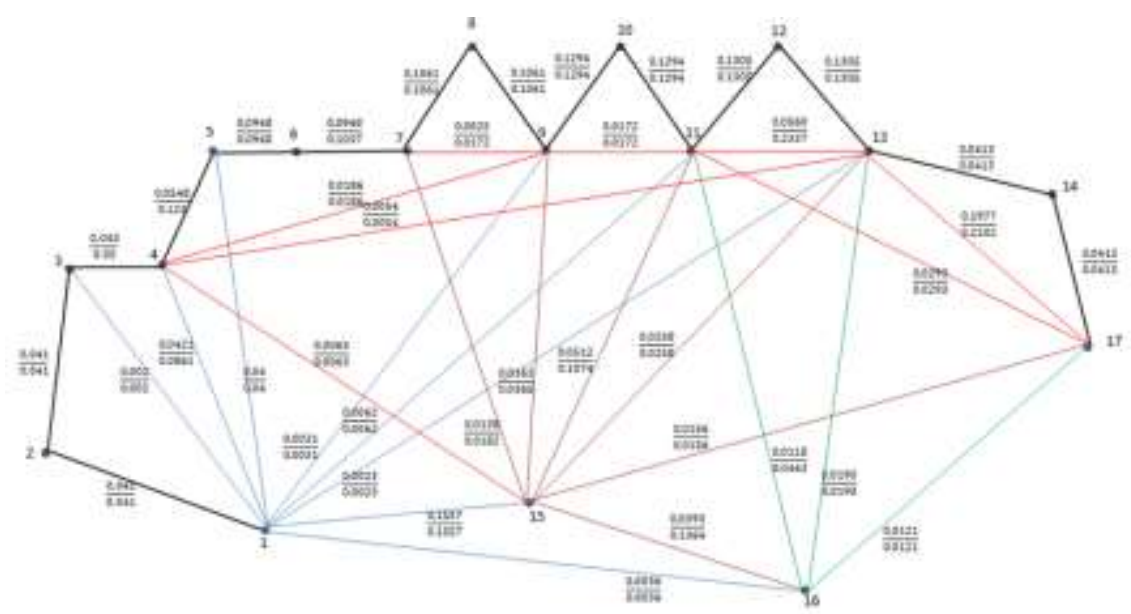

Fig. 12. Graphical solution of the bi-level problem

The comparisons between the solutions of single optimization case on Fig. 10 and the bi-level one from Fig. 12 demonstrate that the bi-level problem increases the usage of the rail transportation. For example:

- Link (7-9) increase from 0.0948 to 1.061 , which is the value of the available capacity of this link. Similar increases are met additionally for a set of rail-supported links.

- Link (9-11) increase from 0.0993 to 0.1294 , which is the value of the available capacity of this link.

- Link (11-13) increase from 0.0623 to 0.1305 , which is its maximal capacity.

The increase of the rail network utilization is accompanied by decreasing the transportation flows of bus links. This decrease is performed on optimal way by the bi-level optimization model:

- Link (1-4) decreases from 0.0861 to 0.0421.

- Link (13-17) decreases from 0.2202 to 0.197 .

- Link (11-15) decreases from 0.0917 to 0.0512 .

However, the bi-level solution contains not only components with decreasing flows through links, operated by buses. For example, the flow distribution through link (11-16) increases from 0.0014 to 0.0118 ; link (15-16) also increase its flow from 0.0289 to 0.0393 . That is why the single and bi-level optimization models have to be assessed and compared under common evaluation criterion. This criterion has been defined taking into consideration that the common goal of the optimization models is to increase the utilization of the rail transportation between two points of the transportation network. That is why this research estimates both the maximal value of the traffic (the value of max flow) which can be transported through the network and the flow distribution of the max flow through the links of the network. The percentage of the max flow, which passes through links, operated by rail transport will be a criterion for assessment the benefit from the different optimization models. Hence, the model, which provides bigger part of the max flow for rail, is preferable for the management of the rail transport. 
The comparisons and assessments between the single and bi-level optimization are evaluated quantitatively in a following way. The total capacity of the rail transportation is defined as a sum of all capacities of links, implemented by rail transport or

TOTAL $\mathrm{L}_{\text {rail }}=v_{1,2}+v_{2,3}+v_{3,4}+v_{4,5}+v_{5,6}+v_{6,7}+v_{7,8,9}+v_{9,10,11}+v_{11,12,13}+v_{13,14}+v_{14,17}=0.8198$ [relative capacity].

The part of the max flow, which passes through the links, operated by rails are evaluated as

Part_MAXF_LOW ${ }_{\text {rail }}=f_{1,2}+f_{2,3}+f_{3,4}+f_{4,5}+f_{5,6}+f_{6,7}+f_{7,8,9}+f_{9,10,11}+f_{11,12,13}+f_{13,14}+f_{14,17}$ [relative capacity].

The flow solution $f_{i, j}, i, j=1, \ldots, N$ for the single and bi-level optimization models are evaluated as

Part_MAXF_LOW rail (single optimization $)=0.6046$,

Part_MAXF_LOW rail (bi-level optimization $)=0.7357$.

The relative transportation of the components of the max flow for both models are:

$$
\begin{aligned}
& \frac{\text { Part_MAXF_LOWrail(single optimization) }}{\text { TOTALrail }} 73.74 \% \text {, } \\
& \frac{\text { Part_MAXF_LOWrail(bi-level optimization) }}{\text { TOTALrail }}=89.74 \% \text {. }
\end{aligned}
$$

This comparison gives advantages for the application of the bi-level optimization models. The total value of the max flow is the same for both models, but the bi-level gives considerable priority for the utilization of the rail transport, Fig. 13.

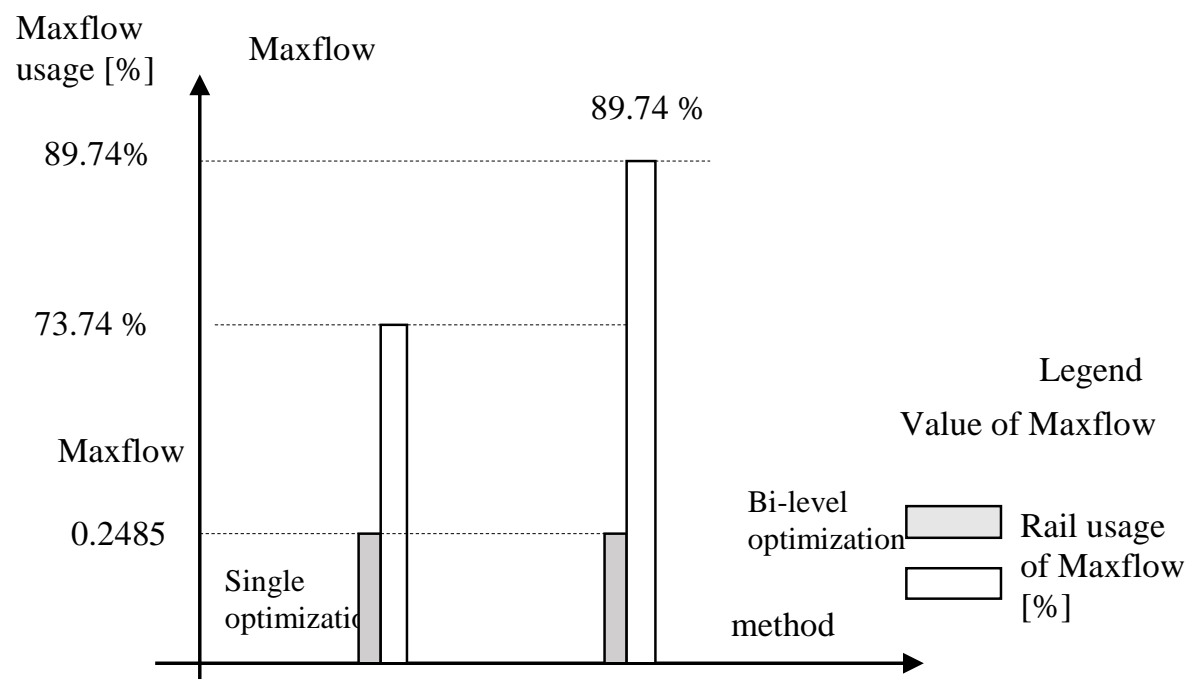

Fig. 13. Comparisons and benefits from the bi-level optimization model

These results prove that the bi-level optimization model gives additional benefits for the rail transport by maximization of the flow between two nodes of the 
network and simultaneously providing priority to the rail transport by redirecting the flows to rail transport, which support low cost flow transport.

\section{Conclusions}

This research presents a new application of the bi-level formalism, modeling and optimization. It has been defined a real optimization problem which gives solutions increasing the exploitation and movement by rail transport. It gives formal basis for providing policies of transportation authorities to issue licensees for bus transportation. The formal model for increasing the rail transport founds on the integration of optimization problems for maximization of the transport flow between two points of the transport network. Additionally, advantages are given to the rail transport by applying the problem of flow distribution with minimal costs.

Thus, the bi-level problem gives solution both for rail and bus transportation and takes into consideration requirements for improving and giving priority to rail in a common transportation network. The derived solutions additionally identify a set of weak points, where the rail infrastructure has to be improved by suggesting intensification of rail schedule per link. The parameters of the bi-level problems have been estimated according to the real transportation policy in Bulgaria. The peculiarity of this application of bi-level optimization is the exact definition of the hierarchical optimization problem, which give practical results despite the incomplete data of rail and buses transportation volumes.

A perspective approach for improving the models and solutions by giving priority to rail transport could be the implementation of fuzzy models and soft computing $[2,25]$ and/or multi criteria optimization $[24,26]$ in a multilevel hierarchical problem. By increasing the levels of optimization and the formal content of the problems on different levels, one can expect additional benefits for the rail management. However, currently the modelling using multilevel computation meets practical difficulties because of the increase of the computational time, which constrains the application of the multi-level formalism in practical cases.

Acknowledgement: This work is partly supported by two projects: 134/20.6.2016 with the "BDZ Passenger Services", Ltd, in Bulgaria and No ДФНП-98-A1/04.05.2016, Program for career development of young scientists, Bulgarian Academy of Sciences.

\section{References}

1. A 11 e $\mathrm{n}$ d e, G. B., G. S t I 1 1. Solving Bi-Level Programs with the KKT-Approach. - Math. Program, Ser. A., Vol. 138, 2013, pp. 309-332. DOI 10.1007/s10107-012-0535-x. art\%3A10.1007\%2Fs10107-012-0535-x.pdf

2. A n g e 1 o v a, V. Investigations in the Area of Soft Computing. Targeted State of the Art Report. Cybernetics and Information Technologies, Vol. 9, 2009, No 1, pp. 18-24. http://www.cit.iit.bas.bg/CIT_09/v9-1/18-24.pdf

3. B a r d, J. F. Coordination of Multi-Divisional Firm through Two Levels of Management. - Omega, Vol. 11, 1982, No 5, pp. 457-465.

4. B a r d, J. F. Practical Bilevel Optimization: Algorithms and Applications. The Netherlands, Kluwer, 1998. 
5. C a lve te, H. I., C. G a lé, M. O li v e r o s. Bi-Level Model for Production Distribution Planning Solved by Using Ant Colony Optimization. - Computers and Operations Research, Vol. 38, 2011, No 1, pp. 320-327.

6. C e c c h i n i, M., J. E c k e r, M. K u p f e r s c h m i d, R. L e i t c h. Solving Nonlinear Principalagent Problems Using Bilevel Programming. - European Journal of Operational Research, Vol. 230, 2013, No 2, pp. 364-373.

7. Cols o n, B., P. M a r c o t t e, G. S a v a r d. An Overview of Bi-Level Optimization. - Annals of Operational Research, Vol. 153, 2007, pp. 235-256.

8. D e m p e, S. Annotated Bibliography on Bilevel Programming and Mathematical Programs with Equilibrium Constraints. - Optimization, Vol. 52, 2003, No 3, pp. 339-359.

9. D e m p e, S., V. V. K a l a s h n i k ov, G. A. Pé r e z-V a ld é s, N. K a 1 a s h n y k o va. Natural Gas Bi-Level Cash-Out Problem: Convergence of a Penalty Function Method. - European Journal of Operational Research, Vol. 215, 2011, No 3, pp. 532-538.

10. F a m p a, M., L. B a r r o s o, D. C a n d a 1, L. S i i m o n e t t i. Bi-Level Optimization Applied to Strategic Pricing in Competitive Electricity Markets. - Computational Optimization and Applications, Vol. 39, 2008, No 2, pp.121-142.

11. F a n g, S., P. Gu o, M. Li, L. Z h a n g. Bi-Level Multi-Objective Programming Applied to Water Resources Allocation. - Mathematical Problems in Engineering, Vol. 2013, 2013, Article ID 837919. 9 p.

12. F o r d, L. R. Jr., D. R. F u 1 k e r s o n. Maximal Flow through a Network. - Canadian Journal of Mathematics, Vol. 8, 1956, pp. 399-404. DOI:10.4153/cjm-1956-045-5, MR 0079251

13. G o m o r y, R. E., T. C. H u. Multi-Terminal Network Flows. - Journal of the Society for Industrial and Applied Mathematics, Vol. 9, December 1961, No 4, pp. 551-570.

14. H a r r i s, T. E., F. S. R o s s. Fundamentals of a Method for Evaluating Rail Net Capacities. (U), RM-1 573, 24 October, 1955.

15. H o, H. W., S. C. W o n g. Housing Allocation Problem in a Continuum Transportation System. Transportmetrica, Vol. 3, 2007, No 1, pp. 21-39.

16. K a l a shnikov, V., S. D e m p e, G. A. Pér e z-V a ld és, N. K a l a s hn y k ova, J. F. $\mathrm{C}$ a $\mathrm{m}$ a c h o-V a $11 \mathrm{e} \mathrm{j}$ o. Bilevel Programming and Applications. Review Article. Mathematical Problems in Engineering, Vol. 2015, 2015, Article ID 310301. 16 p. http://dx.doi.org/10.1155/2015/310301

17. K a r a, B. Y., V. V e r t e r. Designing a Road Network for Hazardous Materials Transportation. Transportation Science, Vol. 38, 2004, pp. 188-196.

18. K h e i r k h a h, A., N. H a m i d R e z a, M. B. M a s u m e. A Bi-Level Network Interdiction Model for Solving the Hazmat Routing Problem. - Special Issue: Transportation in Supply Chain Management, Vol. 54, 2016, Issue 2, pp. 459-471. http://dx.doi.org/10.1080/00207543.2015.1084061

19. K k a y d i n, Hand e, N e c a t i A r a s, I. K u b a n A l t n e l. Competitive Facility Location Problem with Attractiveness Adjustment of the Follower: A Bilevel Programming Model and Its Solution. - European Journal of Operational Research, Vol. 208, 2011, No 3, pp. 206-220.

20. K o v a c e vi c, R., G. Ch. P f 1 u g. Electricity Swing Option Pricing by Stochastic Bilevel Optimization: A Survey and New Approaches. - European Journal of Operational Research, Vol. 237, 2014, Issue 2, pp. 389-403.

21. K ü c ü k a y d i n, H., N. A r a s, I. K. A $1 \mathrm{t}$ i n e 1. Competitive Facility Location Problem with Attractiveness Adjustment of the Follower: A Bi-Level Programming Model and Its Solution. - European Journal of Operational Research, Vol. 208, 2011, No 3, pp. 206-220.

22. M o r t o n, D., F. P a n, K. S a e g e r. Models for Nuclear Smuggling Interdiction. - IIE Transactions, Vol. 39, 2007, No 1, pp. 3-14.

23. O u t r a t a, J., M. K o c v a r a. Effective Reformulations of the Truss Topology Design Problem. - Optimization and Engineering, Vol. 7, 2006, pp. 201-219.

24. P e n e v a V., I. P o p c h e v. Multicriteria Decision Making Based on Fuzzy Relations. - Cybernetics and Information Technologies, Vol. 8, 2008, No 4, pp. 3-12.

25. P e n e v a, V., I. P o p c h e v. Multicriteria Decision Making by Fuzzy Relations and Weighting Functions for the Criteria. - Cybernetics and Information Technologies, Vol. 9, 2009, No 4, pp. 58-41. 
26. R a d e v a, I. Multicriteria Models for Clusters Design. - Cybernetics and Information Technologies, Vol. 13, 2013, No 1, pp. 18-33.

http://www.cit.iit.bas.bg/CIT_2013/v13-1/Radeva.pdf

27. S c h r i j v e r, A. On the History of the Transportation and Maximum Flow Problems. - Documenta Mathematica, Extra Volume ISMP, 2012, pp. 169-180.

http://www.math.uiuc.edu/documenta/vol-ismp/33_schrijver-alexander-tmf.pdf

28. S t o i $10 \mathrm{v}$, T., K. S t o i 1 o v a, M. P a p a g e or g i o u, I. P a p a m i c h a i 1. Bi-Level Optimization in a Transport Network. - Cybernetics and Information Technologies, Vol. 15, 2015, No 5, pp. 37-49.

29. S t o i 1 o v a, K., T. S t o i 1 o v, K. N i k o 1 o v. Autonomic Properties in Traffic Control. Cybernetics and Information Technologies, Vol. 13, 2013, No 4, pp. 18-32.

30. S u n, H., Z. G a o, J. W u. A Bi-Level Programming Model and Solution Algorithm for the Location of Logistics Distribution Centers. - Applied Mathematical Modelling, Vol. 32, 2008, No 4, pp. 610-616.

31. V i c e n t e, L. N., P. H. C a 1 a m a i. Bilevel and Multilevel Programming: A Bibliography Review. - Journal of Global Optimization, Vol. 5, 2004, No 3, pp. 291-306.

32. W a n g, F. J., J. P e r i a u x. Multi-Point Optimization Using Gas and Nash/Stackelberg Games for High Lift Multi-Airfoil Design in Aerodynamics. - In: Proc. of Congress on Evolutionary Computation (CEC'2001), 2001, pp. 552-559.

33. X u, J., Y. T u, Z. Z e n g. Bi-Level Optimization of Regional Water Resources Allocation Problem under Fuzzy Random Environment. - Journal of Water Resources Planning and Management, Vol. 139, 2013, No 3, pp. 246-264.

34. https://yalmip.github.io/command/solvebilevel/ 